\title{
HOT CORE CHEMISTRY: GAS PHASE MOLECULE FORMATION IN SITU.
}

\author{
S.B. CHARNLEY ${ }^{\dagger}$ and A.G.G.M. TIELENS \\ Space Science Division, NASA Ames Research Center, California 94035.
}

\begin{abstract}
The possibility that the observed abundances of several molecules in the Orion Hot Core are due to gas phase neutral chemistry has been examined.
\end{abstract}

The Orion Hot Core is a high temperature $(200-300 \mathrm{~K})$, dense $\left(n\left(\mathrm{H}_{2}\right) \sim 10^{7} \mathrm{~cm}^{-3}\right)$ clump of gas. It may represent a state of interstellar gas which is common to star-forming regions. The chemistry of the Hot Core is markedly different from that observed cold molecular clouds and also from that of nearby sources within OMC-1 itself ( Blake et al. 1987; Lacy et al. 1989; Turner 1991). It is believed that the Hot Core is a region in which molecular ice mantles have recently been evaporated from warm dust grains. Brown, Charnley \& Millar (1988) presented a simple hot core theory which qualitatively reproduced the observed composition. In this picture the presence of a given molecule in a hot core can be accounted for in one of three ways. It may either 1) be formed by cold chemistry during isothermal collapse, accreted at its abundance at the free-fall time, and subsequently released unaltered from the mantle in the hot core, or 2) be formed by grain surface chemistry between accreted atoms and molecules, or 3) be formed in situ by neutral-neutral chemistry in the hot gas by reactions involving radicals (present due to a 'burst' of ion-molecule chemistry following mantle evaporation) such as $\mathrm{OH}, \mathrm{CN}, \mathrm{NH}_{2}$ and $\mathrm{CH}_{3}$, and mantle molecules. Starting with the simplest mantle compositions, we have attempted to constrain what reactions may have occurred on grain surfaces, and also the original chemical composition of the cold phase, by first examining the plausibility of the thesis that several molecules are produced in situ. We have modelled the neutral chemistry which ensues following the evaporation of ice mantles containing various ratios of the composition $\mathrm{H}_{2} \mathrm{O}: \mathrm{NH}_{3}: \mathrm{CH}_{4}: \mathrm{CO}: \mathrm{HCN}: \mathrm{HNC}: \mathrm{C}_{2} \mathrm{H}_{2}: \mathrm{H}_{2} \mathrm{CO}: \mathrm{C}_{2} \mathrm{H}_{4}$ into gas with physical conditions similar to that found in Orion. Figure 1 shows the chemical evolution in a representative model.

The original model of Brown et al. produced too much HNC and insufficient HCN due to the accretion of gas with a cold cloud $\mathrm{HCN} / \mathrm{HNC}$ ratio $(\sim 1)$. It was postulated that the conversion of $\mathrm{HNC}$ to $\mathrm{HCN}$ by $\mathrm{H}$ atom reaction may occur on the dust. This reaction probably has a large barrier in the solid state; we have included it as a gas phase process with a barrier of $100 \mathrm{~K}$ as suggested by Pineau des Forêts et al. (1990) and find that this, not unexpectedly, leads to HCN/HNC ratios more in accord with observation. The calculated abundance of $\mathrm{CN}$ in the models is always below the observed upper limit $\left(<5 \times 10^{-10}\right)$.

The reaction of $\mathrm{CN}$ with acetylene to form $\mathrm{HC}_{3} \mathrm{~N}$ will proceed rapidly hot core temperatures (Lichtin \& Lin 1986). The observed range of estimates for the abundance of acteylene (Evans et al. 1991) are sufficient to produce large abundances of this molecule. Brown et al. showed that the hot core abundance of $\mathrm{HC}_{3} \mathrm{~N}$ could be explained by cold phase chemistry. It is likely that the presence of other cyanopolyynes in the Orion Hot Core (e.g. $\mathrm{HC}_{5} \mathrm{~N}$ and $\mathrm{HC}_{7} \mathrm{~N}$, Turner 1991), which exhibit similar cold phase evolution, is also due to freeze-out of cold phase abundances. Herbst \& Leung (1990) have postulated that the reaction of CN with ethene has a significant channel leading to the formation of vinyl cyanide $\left(\mathrm{CH}_{2} \mathrm{CHCN}\right)$, if so, it will be rapid at hot core temperatures (Lichtin \& Lin 1986). Reproduction of the observed vinyl cyanide abundance requires ethene

†NAS/NRC Resident Research Associate 
to be present in the mantles at an abundance similar to that of acetylene. Hydrogenation of accreted acetylene to ethene and ethane may be important on grain surfaces (Tielens 1991). We predict that $\mathrm{C}_{2} \mathrm{H}_{4}$ is present in the Hot Core and that $\mathrm{C}_{2} \mathrm{H}_{6}$ and should also be present. Reaction of $\mathrm{CN}$ with $\mathrm{C}_{2} \mathrm{H}_{6}$ proceeds rapidly at $300 \mathrm{~K}$ but the products probably do not include $\mathrm{CH}_{3} \mathrm{CH}_{2} \mathrm{CN}$ (Lichtin \& Lin 1985); we have been unable to discover a viable neutral chemistry for this molecule. The abundances of $\mathrm{HC}_{3} \mathrm{~N}\left(\sim 2 \times 10^{-9}\right)$ produced by cold phase chemistry (Brown et al. ) are too low to provide a viable source of $\mathrm{CH}_{2} \mathrm{CHCN}$ and $\mathrm{CH}_{3} \mathrm{CH}_{2} \mathrm{CN}$ by grain surface hydrogenation of $\mathrm{HC}_{3} \mathrm{~N}$, as has been suggested (Tielens 1991). Acetylene, $\mathrm{CH}_{3} \mathrm{CN}$ and $\mathrm{H}_{2} \mathrm{CO}$ cannot be efficiently synthesised in the hot gas from the simplest mantle compositions, however, the observed abundances of these molecules can be adequately reproduced by formation in the cold phase. It is not certain whether the NO observed in Orion is located in the Hot Core, in the Compact Ridge, or in both (Ziurys et al. 1991). NO cannot be efficiently formed at the temperature of the Orion Hot Core but it can be at lower temperatures $(\sim 100 \mathrm{~K})$ suggesting that, if formed in situ, it is present in the Compact Ridge. If NO is indeed present in the Hot Core then perhaps the accreted NO is hydrogenated to HNO on grains and, following evaporation, recovered as NO in the hot gas by reaction with $\mathrm{H}$ atoms (Westley 1980).

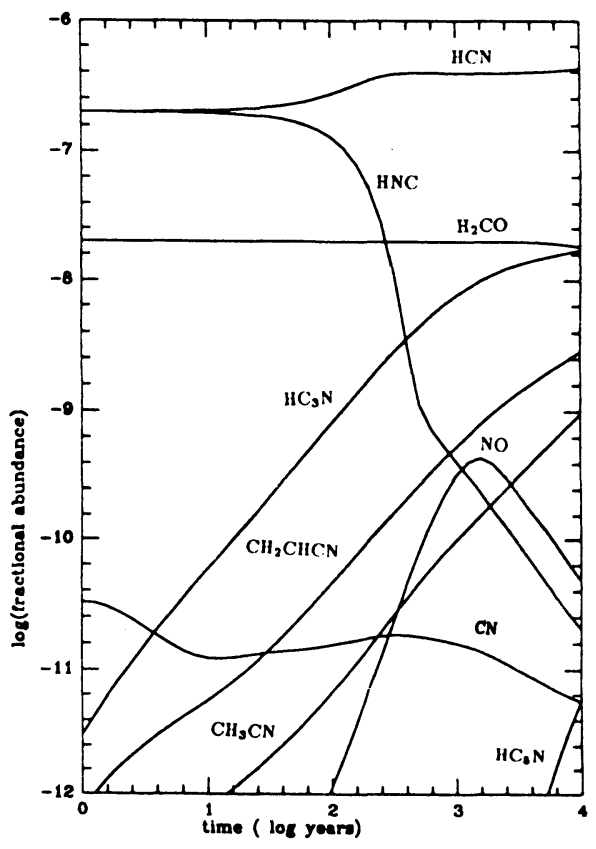

A complex hot core chemistry may be driven by relatively simple mantle compositions. The actual chemistry which occurs depends upon the presence, or otherwise, of certain mantle molecules and the density and temperature of the core gas. This can lead to strong chemical differentiation between cores (Charnley 1991; Charnley, Tielens \& Millar 1991).

Figure 1. Evolution of some neutral molecules in a hot core following mantle evaporation $\left(n\left(\mathrm{H}_{2}\right)=\right.$ $\left.10^{7} \mathrm{~cm}^{-3} ; \mathrm{T}=250 \mathrm{~K}\right)$. The mantle composition was that inferred from observation (see references in text) for water, ammonia, methane, formaldehyde and $\mathrm{CO}$. $\mathrm{HCN}$ and $\mathrm{HNC}$ were assumed to have equal abundances. The largest estimate for the acetylene abundance was used $\left(\sim 10^{-6}\right)$. Ethene was included at an abundance equivalent to that of acetylene.

\section{References}

Blake, G.A., Sutton, E.C., Masson, C.R. \& Phillips, T.G. 1987, Ap.J., 315, 621.

Brown, P.D., Charnley, S.B. \& Millar, T.J. 1988, M.N.R.A.S., 231, 409.

Charnley, S.B. 1991, in preparation.

Charnley, S.B., Tielens, A.G.G.M. \& Millar, T.J. 1991, in preparation.

Evans, N.J., Lacy, J.H. \& Carr, J.S. 1991, preprint.

Herbst, E. \& Leung, C.M. 1990, Astr.Ap., 177, 180.

Lichtin, D.A. \& Lin, M.C. 1985, Chem.Phys. 473, 482.

Lichtin, D.A. \& Lin, M.C. 1986, J.Chem.Phys. 325, 330.

Pineau des Forêts, Roueff, E \& Flower, D. 1990, M.N.R.A.S., 244, 668.

Tielens, A.G.G.M. 1991, in Chemistry and Spectroscopy of Interstellar Molecules,

ed. N. Kaifu, University of Tokyo Press

Turner, B. 1991, Ap.J.Suppl., 76, 617.

Westley, F. 1980, NSRDS-NBS 67; Washington DC.

Ziurys, L., McGonagle, D., Minh, Y. \& Irvine, W.M. 1991, Ap.J., 373, 535. 